\title{
Traitement de matériaux métalliques par choc laser en géométrie confinée à $248 \mathrm{~nm}$
}

\author{
E. Ohayon, Th. Sarnet et M. Autric
}

Institut de Mécanique des Fluides, Equipe Interaction Laser-Matière, Parc Scientifique et Technologique de Luminy, 13009 Marseille, France

\begin{abstract}
: we report here some preliminary results of a study concerning basic physical and metallurgical processes involved in laser-shock treatment of materials. This laser shock processing consists of the use of a high power pulsed $248 \mathrm{~nm}$ excimer laser to generate a short duration, high amplitude pressure which propagates inside various metallic samples, aluminium, titanium and iron alloys.Pressure measurements on the rear side of the sample have been obtained in direct ablation and water-confined plasma regimes.Experimental results are compared with an analytical model.Surface hardness, in-depth residual stresses and fatigue tests have to be compared in both the interaction regimes.
\end{abstract}

\section{INTRODUCTION}

Lorsqu'un rayonnement laser intense irradie un matériau solide placé à l'atmosphère ambiante, un plasma de fortes température, densité et pression se forme à la surface,puis se détend dans le milieu.Ce mode d'interaction (régime d'ablation directe) limite fortement la pression qu'il est possible de générer dans le solide.

Les travaux réalisés précédemment dans cette configuration (principalement couplage mécanique et radiatif) [1] ont été poursuivis par l'étude des phénomènes de couplage rayonnement-matière en géométrie confinée (technique de confinement consistant à empêcher la détente du plasma à l'aide d'un diélectrique et obtenir ainsi des pressions plus importantes). Ce type d'études largement développées au LALP à 1,06 $\mu \mathrm{m}$ [2] [3] a été entreprise à l'IMFM à $248 \mathrm{~nm}$.

\section{CONDITIONS EXPERIMENTALES ET RESULTATS}

Les résultats présentés dans ce papier concernent l'amplitude des pressions mesurées en face arrière de l'échantillon au moyen de jauges de contraintes piézoélectriques au PVDF ainsi que les analyses métallurgiques principalement en termes de microdureté superficielle.Les expériences ont été réalisées sur des alliages à base d'aluminium 2017A (AU4G) et de titane (TA6V) ainsi que sur un acier 35NCD16 sous confinement eau au moyen d'un iuser à excimères $\mathrm{KrF}(248 \mathrm{~nm})$ capable de délivrer $200 \mathrm{~J}$ en $400 \mathrm{~ns}$. Les matériaux ont été irradiés entre 100 et $600 \mathrm{MW} / \mathrm{cm} 2(\tau=350 \mathrm{~ns}$; temps de montée de l'impulsion=90ns ) sur une surface comprise entre 0,1 et $0,6 \mathrm{~cm} 2$. Le seuil de claquage du milieu de confinement n'a pas été atieint.Les pressions obtenues varient entre 10 et $360 \mathrm{MPa}$ derrière $1 \mathrm{~mm}$ de matière. Un rapport d'un facteur 6 a pu être 
observé entre le mode direct et le mode confiné (figure 1).Une comparaison des résultats expérimentaux obtenus avec un modèle analytique développé au LALP a été entreprise :

$$
\mathrm{P}=10 \sqrt{\frac{\alpha}{2 \alpha+3} \sqrt{\mathrm{Z}} \sqrt{\phi}}
$$

où $\alpha$ est un terme correctif, $\mathrm{P}$ la pression en $\mathrm{MPa}, \mathrm{Z}$ l'impédance de choc en $\mathrm{g} . \mathrm{cm}^{-2}$.s- 1 et $\phi$ le flux en $\mathrm{GW} / \mathrm{cm} 2$

Les tirs laser réalisés sur des échantillons détensionnés permettent l'analyse en surface et en profondeur de la mise sous contrainte de ces métaux.Le niveau des contraintes résiduelles créées dans le matériau (fonction de la pression atteinte lors de l'interaction et de son temps de maintien) sera analysé par diffraction de rayons $X$ en mode d'ablation directe et en mode confiné.

Une augmentation significative de la dureté superficielle du $35 \mathrm{NCD} 16$ (figure 2) et du TA6V a également été mise en évidence contrairement à l'AU4G[4]

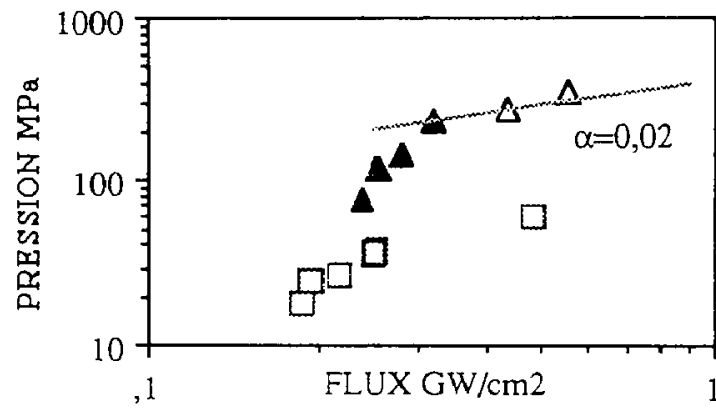

$\triangle$ AU4G + Cuivre confiné e:u

$\Delta$ AU4G confiné eau

AU4G direct

modèle

fig:ıre 1 Comparaison modèle analytique / expérience-AU4G e=1mm

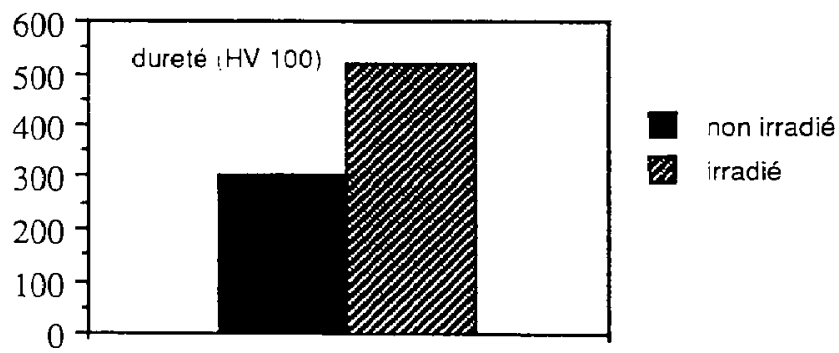

figure 2 Moditication de dureté sur acier 35NCD16 - $0,4 \mathrm{GW} / \mathrm{cm} 2$

\section{Remerciements}

Les auteurs tiennent à remercier le Laboratoire Mecasurf de l'ENSAM Aix en Provence pour leur aide technique dans la caractérisation métallurgique des échantillons.

\section{References}

[1] Montagne J.E., Thèse de doctorat de l'Université d'Aix-Marseille II, "Laser exciplexes $\mathrm{KrF}$ impulsionnel de grande energie-Effets du rayonnement ultra-violet sur matériaux". Dec 1992

[2]Fabbro R., Fournier J.. Ballard P., Devaux D., Virmont J., "Physical study of laser -produced plasma in contined geometry" J.Appl.Phys. 68(2), 15 July 1990 pp 775-784

[3]Devaux D., Fabbro R., Tollier L., Bartnicki E., "Generation of shock waves by laser -induced plasma in confined geometry" J.Appl.Phys.74 (4), 15 August 1993 pp 2268- 2273

[4]Sarnet Th., Montagne J.E.. Inglesakis G., Autric M., Barralier L., Barreau G., "Modification of metals by high energy excimer laser" Journal de physique 4, 4 April 1994 pp $69-72$ 